\title{
Versuche zur Synthese des Cytisins. Dehydrierung von Tetrahydro-cytisin zu Cytisin.
}

\author{
Von
}

\begin{abstract}
F. Galinovsky, 0. Vogl* und W. Moroz.
Aus dem II. Chemischen Laboratorium der Universität Wien.

(Eingelangt am 8. Juli 1954.)

Die Dehydrierung von Tetrahydro-cytisin zu Cytisin mit Palladium verläuft glatt, wenn man die Iminogruppe durch Acetylierung schützt. Durch Erhitzen des N-Acetyl-tetrahydrocytisins mit $\mathrm{Pd}-\mathrm{Mohr}$ bei $280^{\circ}$ erhält man so in guter Ausbeute N-Acetyl-cytisin und daraus durch Abspaltung der Acetylgruppe mit Salzsäure das Cytisin.
\end{abstract}

Wir haben vor einiger Zeit über die Synthese des Tetrahydro-desoxycytisins (I) berichtet ${ }^{1}$, welches das vollständige tricyclische Ringsystem des Cytisins (IV) enthält. Durch Spaltung der racemischen Verbindung mit Weinsäure konnte der rechtsdrehende Antipode erhalten werden, der mit der aus Cytisin durch Perhydrierung des $\alpha$-Pyridonringes erhaltenen Verbindung identisch war. Wenn man vom Tetrahydro-desoxycytisin (I) ausgeht, so ist zur Vollendung der Synthese des Cytisins die Umwandlung des Ringes $\mathbf{A}$ in einen $\alpha$-Pyridonring notwendig, die in zwei Stufen durchgeführt werden kann. Durch Einführung eines Sauerstoffatoms in den Ring $\mathbf{A}$ in $\alpha$-Stellung zum bicyclisch gebundenen Stickstoff würde man zum Tetrahydro-cytisin (II) und durch Dehydrierung des letzteren zum Cytisin (IV) selbst gelangen. Zur Darstellung des Tetrahydro-cytisins sind natürlich auch andere Synthesewege denkbar. Ein den Autoren als eventuell realisierbar erscheinender Weg wurde kürzlich von Bohlmann, Ottawa und Keller ${ }^{2}$ angegeben. In dieser Arbeit angekündigte Versuche zur Dehydrierung des Tetrahydro-cytisins ver-

* Derzeitige Adresse: University of Michigan, Department of Chemistry, Ann Arbor, Michigan, USA.

${ }^{1}$ F. Galinovsky, O. Vogl und W. Moroz, Mh. Chem. 83, 242 (1952).

2 F. Bohlmann, N. Ottawa und R. Keller, Ann. Chem. 587, 162 (1954). 
anlassen uns nun, unsere eigenen schon vor einiger Zeit mit dieser Verbindung erfolgreich durchgeführten und in einer Dissertation ${ }^{3}$ niedergelegten Dehydrierungsversuche noch vor Abschluß unseres weiteren Versuchsprogramms zu veröffentlichen.<smiles>[B]N1CCCC2CCCCC21</smiles>

I<smiles>[Y]N1C[C@@H]2C[C@H](C1)[C@@H]1CCCC(=O)N21</smiles>

II: $\mathrm{R}=\mathrm{H}$

III: $\mathrm{R}=\mathrm{OC} \cdot \mathrm{CH}_{3}$<smiles>[R]N1C[C@@H]2C[C@H](C1)c1cccc(=O)n1C2</smiles>

$\mathrm{IV}: \mathrm{R}=\mathrm{H}$

$\mathrm{V}: \mathrm{R}=\mathrm{OC} \cdot \mathrm{CH}_{3}$

Wie schon von Späth und Galinovsky $y^{4}$ gefunden wurde, verläuft die Dehydrierung vieler hydrierter $\alpha$-Pyridonderivate mit Pd-Mohr bei Temperaturen von 240 bis $270^{\circ}$ ziemlich glatt unter Bildung der entsprechenden $\alpha$-Pyridonderivate. Es bot aber trotzdem wenig Aussicht auf Erfolg, vom Tetrahydro-cytisin durch Dehydrierung mit Palladium zum Cytisin zu gelangen, da dieses beim Erhitzen mit Palladium zur Hauptsache eine Aufsprengung des Ringes C, der den sekundären Stickstoff enthält, erleidet, wobei ein komplexes Gemisch von Reaktionsprodukten entsteht, aus dem schon früher durch Umlagerung entstandene Verbindungen, wie 2-0xy-6-methyl-chinolin, isoliert werden konnten ${ }^{5}$. Auch andere Versuche zur Dehydrierung des Tetrahydro-cytisins zum Cytisin blieben erfolglos. Es wurde nun die Dehydrierung eines Derivates des Tetrahydro-cytisins versucht, das die Iminogruppe geschützt enthält. Das bei $164^{\circ}$ schmelzende N-Acetyl-tetrahydro-cytisin (III) erwies sich als ausgezeichnet brauchbar. Nach 4stündigem Erhitzen mit Pd-Mohr bei einer Höchsttemperatur von $280^{\circ}$ (Metallbad) wurde bei der folgenden Destillation im Hochvak. eine Fraktion erhalten, die sofort kristallisierte und aus der sich in zirka 60\%iger Ausbeute reines N-Acetyl-cytisin (V) isolieren ließ. Durch Erhitzen mit Salzsäure wurde daraus quantitativ das Cytisin (IV) selbst erhalten. Diese einfache Methode zur Einführung der Doppelbindungen in den Piperidonring des Tetrahydro-cytisins macht im Hinblick auf einen Abschluß der Synthese des Cytisins die synthetische Darstellung des Tetrahydro-cytisins besonders wünschenswert.

3 W. Moroz, Dissertation Universität Wien (1953).

4 Ber. dtsch. chem. Ges. 69, 2059 (1936).

5 F'. Breusch, Dissertation Universität Wien (1927). -.. E. Späth und F. Galinovsky, Ber. dtsch. chem. Ges. 69, 761 (1936). 


\author{
Experimenteller Teil. \\ $N$-Acetyl-tetrahydro-cytisin (III).
}

Tetrahydro-cytisin (II) wurde durch katalytische Hydrierung des Cytisins, wie früher beschrieben, dargestellt, nur wurde statt Pd-Tierkohle $\mathrm{PtO}_{2}$ nach Adams als Katalysator genommen (ein Zehntel der Gewichtsmenge des Cytisins) und in wäßr. Lösung hydriert, wobei die Hydrierung nach Aufnahme von 2 Molen $\mathrm{H}_{2}$ nur mehr langsam weiterging. Eine Reduktion der CO-Gruppe trat unter diesen Bedingungen nur in untergeordnetem Maß ein. Die aus Aceton-Äther umgelöste Substanz schmolz bei $114^{\circ}$.

$N$-Acetylverbindung (III): $3 \mathrm{~g}$ II wurden unter Kühlung mit $2 \mathrm{ml}$ Essigsäureanhydrid versetzt, $6 \mathrm{Stdn}$. stehen gelassen, das Anhydrid mit Wasser zersetzt und die sodaalkalisch gemachte Lösung mit Äther erschöpfend extrahiert. Der Ätherrückstand kristallisierte, sublimierte bei 0,01 Torr bei $140^{\circ}$ Luftbadtemp. und schmolz nach dem Umlösen aus Aceton-Äther bei $164^{\circ}$.

$$
\mathrm{C}_{13} \mathrm{H}_{20} \mathrm{O}_{2} \mathrm{~N}_{2} \text {. Ber. C 66,07, } \mathrm{H} 8,53 \text {. Gef. C 65,79, H 8,56. }
$$

Dehydrierung des $N$-Acetyl-tetrahydro-cytisins (III) zu N-Acetyl-cytisin (V).

$0,30 \mathrm{~g}$ III wurden mit $0,21 \mathrm{~g} \mathrm{Pd}-\mathrm{Mohr}$ gut vermengt und im Kugelrohr 4 Stdn. im Metallbad bei 250 bis $280^{\circ}$ erhitzt, wobei die Temp. langsam gesteigert wurde. Nach Beendigung der Dehydrierung wurde zuerst eine geringe Menge Vorlauf durch Destillation im Wasserstrahlvak. bis $150^{\circ}$ abgetrennt und darauf die Hauptmenge bei 180 bis $190^{\circ}$ Luftbadtemp. und 0,01 Torr destilliert. Das Destillat kristallisierte und wurde aus AcetonÄther umgelöst. Es wurden so $0,17 \mathrm{~g}$ reines Kristallisat erhalten, das die van de Moersche Reaktion mit $\mathrm{FeCl}_{3}$ gab, die für den $\alpha$-Pyridonring charakteristisch ist. Der Schmp. lag bei 209 bis $210^{\circ}$, der Mischschmp. mit bei 210 bis $211^{\circ}$ schmelzendem $\mathrm{N}$-Acetyl-cytisin ${ }^{8}$ bei $210^{\circ}$. Aus der stark eingeengten Mutterlauge konnte noch etwas Ausgangsmaterial erhalten werden.

$$
\begin{gathered}
\text { Drehung von } V: \alpha_{\mathrm{D}}^{18}=-4,43^{\circ}(\text { Wasser, } c=1,69,1-\mathrm{dm}-\text { Rohr }) ;[\alpha]_{\mathrm{D}}^{18}= \\
=-262,1^{\circ} .
\end{gathered}
$$

\title{
Abspaltung der Acetylgruppe.
}

$0,10 \mathrm{~g} \mathrm{~V}$ wurden in $10 \mathrm{ml}$ Salzsäure (1:1) 3 Stdn. am Rückfluß erhitzt. Die Lösung wurde dann im Vak. eingedampft, der Rückstand in wenig Wasser aufgenommen, stark alkalisch gemacht und mit Chloroform erschöpfend. ausgezogen. Das erhaltene Cytisin (IV) wog $0,08 \mathrm{~g}$ und schmolz nach dem Umlösen aus Aceton- ̈̈ther bei 153 bis $154^{\circ}$, der Mischschmp. mit natürlichem Cytisin lag bei der gleichen Temp.

Die C,H-Analyse wurde von Herrn Dr. G. Kainz im Mikrolaboratorium des II. Chemischen Institutes ausgeführt.

6 E. Späth und F. Galinovsky, Ber. dtsch. chem. Ges. 65, 1526 (1932).

7 F. Galinoesky und E. Stern, Ber. dtsch. chem. Ges. 77, 132 (1944).

8 K. Buchka und A. Magalhaes, Ber. dtsch. chem. Ges. 24, 678 (1891). A. Partheil, Arch. Pharmaz. 230, 490 (1892). 